\title{
ARTICLE
}

Myelodysplastic syndrome

\section{S100A9-induced overexpression of PD-1/PD-L1 contributes to ineffective hematopoiesis in myelodysplastic syndromes}

\author{
Pinyang Cheng ${ }^{1} \cdot$ Erika A. Eksioglu $\mathbb{D}^{1} \cdot{ }^{1}$ Xianghong Chen ${ }^{1} \cdot$ Wendy Kandell ${ }^{2}$ Thu Le Trinh ${ }^{1} \cdot$ Ling Cen $^{3} \cdot$ Jin $\mathbf{Q i}^{1}$. \\ David A. Sallman ${ }^{4} \cdot$ Yu Zhang ${ }^{1} \cdot$ Nhan Tu$^{1} \cdot$ William A. Adams $^{1} \cdot$ Chunze Zhang ${ }^{1} \cdot$ Jinhong Liu ${ }^{1} \cdot$ John L. Cleveland ${ }^{5}$. \\ Alan F. List ${ }^{3} \cdot$ Sheng Wei ${ }^{1}$
}

Received: 10 May 2018 / Revised: 4 January 2019 / Accepted: 15 January 2019 / Published online: 8 February 2019

(c) The Author(s) 2019. This article is published with open access

\begin{abstract}
Myelodysplastic syndromes (MDS) are characterized by dysplastic and ineffective hematopoiesis that can result from aberrant expansion and activation of myeloid-derived suppressor cells (MDSCs) within the bone marrow (BM) niche. MDSCs produce S100A9, which mediates premature death of hematopoietic stem and progenitor cells (HSPCs). The PD-1/ PD-L1 immune checkpoint impairs immune responses by inducing T-cell exhaustion and apoptosis, but its role in MDS is uncharacterized. Here we report an increased expression of PD-1 on HSPCs and PD-L1 on MDSCs in MDS versus healthy donors, and that this checkpoint is also activated in S100A9 transgenic (S100A9Tg) mice, and by treatment of BM mononuclear cells (BM-MNC) with S100A9. Further, MDS BM-MNC treated with recombinant PD-L1 underwent cell death, suggesting that the PD-1/PD-L1 interaction contributes to HSPC death in MDS. In accordance with this notion, PD-1/ PD-L1 blockade restores effective hematopoiesis and improves colony-forming capacity in BM-MNC from MDS patients. Similar findings were observed in aged S100A9Tg mice. Finally, we demonstrate that c-Myc is required for S100A9induced upregulation of PD-1/PD-L1, and that treatment of MDS HSPCs with anti-PD-1 antibody suppresses the expression of Myc target genes and increases the expression of hematopoietic pathway genes. We conclude anti-PD-1/anti-PD-L1 blocking strategies offer therapeutic promise in MDS in restoring effective hematopoiesis.
\end{abstract}

Supplementary information The online version of this article (https:// doi.org/10.1038/s41375-019-0397-9) contains supplementary material, which is available to authorized users.

$\triangle$ Sheng Wei

sheng.wei@moffitt.org

1 Department of Immunology, H. Lee Moffitt Cancer Center \& Research Institute, Tampa, FL, USA

2 Cancer Biology PhD Program, University of South Florida and H. Lee Moffitt Cancer Center and Research Institute, Tampa, FL, USA

3 Bioinformatics Core, H. Lee Moffitt Cancer Center, Tampa, FL, USA

4 Department of Malignant Hematology, H. Lee Moffitt Cancer Center \& Research Institute, Tampa, FL, USA

5 Department of Tumor Biology, H. Lee Moffitt Cancer Center \& Research Institute, Tampa, FL, USA

\section{Introduction}

Myelodysplastic syndromes (MDS) are age-dependent hematopoietic stem cell malignancies characterized by dysplastic and ineffective hematopoiesis that result from abnormal and repressed bone marrow (BM) maturation [1-3]. Chronic inflammation coupled with senescence-dependent changes in both hematopoietic stem and progenitor cells (HSPCs) and the BM microenvironment are hallmarks of MDS pathogenesis [4-6]. In particular, excess generation of inflammatory cytokines, the expansion of regulatory $\mathrm{T}$ cells (Tregs), and upregulation or hyperactivation of immune receptors have been shown to drive the development of MDS [7-10]. Moreover, hematopoietic-inhibitory, myeloid-derived suppressor cells (MDSCs) are aberrantly expanded within the $\mathrm{BM}$ and are a paracrine source of S100A9, a proinflammatory protein and a damage-associated molecular pattern [9]. Notably, S100A9 induces expansion and activation of MDSCs, triggers cell death of HSPCs and myeloid and erythroid progenitors, and contributes to ineffective hematopoiesis [9, 10]. Finally, S100A9 transgenic mice (S100A9Tg) 
display age-dependent, excessive accumulation of MDSCs within the BM niche, and develop progressive cytopenias and multilineage cytological dysplasia. Thus, forced expression of S100A9 in vivo is sufficient to provoke hematopoietic alterations that phenocopy human MDS [9, 10].

The immune checkpoint receptor programmed cell death protein-1 (PD-1) and its ligand programmed cell deathligand 1 (PD-L1) have emerged as important regulators of immune system homeostasis. PD-1 is expressed on the surface of activated $\mathrm{T}$ cells, whereas PD-L1 is predominantly expressed on antigen-presenting cells, but also on a variety of other immune cell types. PD-L1 expression is induced during inflammation and by tumor cells within the tumor microenvironment (TME) where it impairs the antitumor immune response [11]. Specifically, engagement of PD-L1 with PD-1 triggers a cascade of events that culminates in exhaustion/dysfunction of activated $\mathrm{T}$ cells $[12,13]$. Germane to our studies, abnormal expression of PD-1 and PD-L1 has been implicated in MDS, where patients treated with azanucleosides and hypomethylating agents have shown an increased expression of checkpoint proteins post treatment [14-16]. Despite these findings, the strong association between inflammation and aging in MDS, and the regulation of the PD-1/PD-L1 axis, the role of these proteins in MDS pathobiology is unclear and unexplored.

Here we report a novel role for the PD-1/PD-L1 pathway in MDS beyond the regulation of T-cell biology, whereby aberrant activation of PD-1 on HSPCs by MDSC-derived PD-L1 triggers hematopoietic cell death, contributes to BM failure, and suppresses hematopoiesis. Most importantly, these data provide direct evidence that PD-1/PD-L1 blockade therapy, alone or in combination with other strategies, may have therapeutic potential to restore and promote effective hematopoiesis in MDS.

\section{Materials and methods}

\section{MDS patients and human BM sample preparation}

All patients were recruited from the Malignant Hematology clinic at the H. Lee Moffitt Cancer Center \& Research Institute. After obtaining written informed consent, patients were classified according to World Health Organization and International Prognostic Scoring System criteria. The specimens used in this research were de-identified save for disease risk as shown in Supplementary Table S1. BM-mononuclear cells (MNC) were isolated from heparinized MDS BM and from heparinized BM of healthy human donors (purchased from Lonza-Walkersville). BM aspirates were first diluted twice their volume in $1 \times$ PBS. BM-MNC were then isolated by Ficoll-Hypaque gradient centrifugation as described [17]. After three washing steps, cells were maintained at $37{ }^{\circ} \mathrm{C}$ in $5 \% \mathrm{CO}_{2}$-humidified atmosphere and cultured in RPMI-1640 medium supplemented with $10 \%$ FBS, $1 \%$ penicillin/streptomycin, and $1 \%$ L-glutamine (Gibco). For some experiments, fresh autologous plasma was used to stimulate BM-MNC $(n=5)$ at $20 \%$ $\mathrm{v} / \mathrm{v}$ for $48 \mathrm{~h}$.

\section{Animals}

Wild-type FVB/NJ mice were purchased from Jackson Laboratory. S100A9Tg mice have been described [9]. Heterozygous c-Myc $\left(\mathrm{Myc}^{+-}\right)$mice were derived from targeted embryonic stem cells $[18,19]$ and were backcrossed onto a pure $\mathrm{C} 57 \mathrm{BL} / 6 \mathrm{~J}$ gene background for more than 15 generations. All mice were housed in pathogenfree units at the Division of Comparative Medicine vivarium at $\mathrm{H}$. Lee Moffitt Cancer Center \& Research Institute/University of South Florida. All animal experiments were approved by the Institutional Animal Care and Use Committee and performed in accordance with U.S. Public Health Service policy and National Research Council guidelines.

\section{Preparation of mouse BM cells}

Each experimental cohort included 5-10 mice. Young and old mice were between 3-4 and 14-16 months old, respectively. Mouse BM cells were obtained from femurs and tibias, red blood cells were removed by ACK lysis buffer (Thermo Fisher Scientific), and BM cells were suspended in PBS with $2 \%$ bovine serum albumin (BSA).

\section{Mouse anti-PD-1 and anti-PD-L1 treatment}

For in vivo anti-PD-1 blockade, the InVivoMAb antimouse PD-1 antibody was acquired from Bio X Cell. Nine-month-old S100A9Tg and age-matched wild-type $\mathrm{FVB} / \mathrm{NJ}$ mice were administered $150 \mu \mathrm{g}$ by intraperitoneal injection twice a week for 6 weeks; $\operatorname{IgG} 2 \mathrm{a}$ was used as a control. Complete peripheral blood counts were performed using a ProCyte Dx Hematology Analyzer according to the manufacturer's protocol (IDEXX Laboratories). Mice were killed at the end of 6-week treatment, and BM cells were collected and used for flow cytometry analyses and colony formation assays.

\section{Flow cytometry}

BM-MNC $\left(1.5 \times 10^{6}\right.$ cells/sample $)$ were stained with appropriate conjugated antibodies in PBS with $2 \%$ BSA. Specifically, human BM-MNC were stained with anti- 
CD33-PE-Cy7, anti-CD34-PerCP-Cy5.5, anti-CD14BV510, anti-CD71-BV421, anti-CD38-BV711, antiCD235a-BUV395, anti-PD-1-FITC, and anti-PD-L1-APC (BD Biosciences). Gating strategies for the identification of myeloid cells, HSPCs, and erythroid progenitors are shown in Supplementary Figure S1. Mouse BM cells were stained with anti-c-Kit-PerCP-Cy5.5, anti-Sca-1-PE, LinAPC (including anti-CD3 $\varepsilon$, anti-CD11b, anti-CD45R/B220, anti-TER-119, anti-Gr-1), anti-PD-1-BV421, anti-PD-L1BV711, anti-PD-L2-BUV395, and anti-CD16/32-PE-Cy7 (BD Biosciences). Cell viability was determined using nearinfrared live/dead dye (BD Biosciences) and the negative (live cell) population was used for further analysis. Samples were acquired on an LSR II flow cytometer and analyzed using FlowJo 9.9.3 software. Intracellular staining with PE-conjugated anti-active caspase-3 was performed using the Cytofix/Cytoperm ${ }^{\mathrm{TM}}$ protocol following initial cell surface receptor staining (BD Biosciences). For PD-1/PDL1 ligation experiments, 2 million BM-MNC were plated per well in 24-well plates coated with recombinant human PD-L1 $(2 \mu \mathrm{g} / \mathrm{mL})$ for $24 \mathrm{~h}$ at $4{ }^{\circ} \mathrm{C}$.

\section{Western blotting}

Western blotting was performed as described previously [9]. Briefly, cell lysates were prepared by resuspending cell pellets in $1 \% \mathrm{NP}-40,10 \mathrm{mM}$ Tris, $140 \mathrm{mM} \mathrm{NaCl}, 0.1 \mathrm{mM}$ PMSF, $10 \mathrm{mM}$ iodoacetamide, $50 \mathrm{mM} \mathrm{NaF}, 1 \mathrm{mM}$ EDTA, $0.4 \mathrm{mM}$ sodium orthovanadate, $10 \mu \mathrm{g} / \mathrm{mL}$ leupeptin, $10 \mu \mathrm{g} /$ $\mathrm{mL}$ pepstatin, and $10 \mu \mathrm{g} / \mathrm{mL}$ aprotinin and lysing on ice for $30 \mathrm{~min}$. Cell lysates were centrifuged at $12,000 \times g$ for 15 min to remove nuclei and cell debris. Protein concentration of the soluble extracts was determined by using the Bradford protein assay (Bio-Rad). Fifty micrograms of protein (per lane) was separated by $10 \%$ SDS-PAGE and transferred to PVDF membranes, which were probed for indicated antibody: anti-PD-1 and anti-PD-L1 (Cell Signaling Technology); anti-c-Myc (Abcam); and anti-beta-actin (Sigma-Aldrich). Proteins were detected with ECL (GE Healthcare Amersham).

\section{Colony formation assay}

Anti-mouse PD-1 and PD-L1, and anti-human PD-1 and PD-L1 Ultra-LEAFTM purified antibodies for neutralization were purchased from BioLegend. Two million BMMNC were plated per well in 24-well plates and cultured with IgG $(5 \mu \mathrm{g} / \mathrm{mL})$, recombinant human S100A9 (rhS100A9; $5 \mu \mathrm{g} / \mathrm{mL})$, anti-PD-1 $(5 \mu \mathrm{g} / \mathrm{mL})$, or anti-PD-L1 $(5 \mu \mathrm{g} / \mathrm{mL})$. After $48 \mathrm{~h}$, cells were collected and used for colony formation assay. Healthy human donor or MDS patient BM-MNC were plated in duplicate in 35-mm culture dishes $\left(1 \times 10^{5}\right.$ cells/dish $)$ in complete methylcellulose media (Stemcell Technologies). Dishes were incubated at $37^{\circ} \mathrm{C}$ in $5 \% \mathrm{CO}_{2}$ for $\sim 10-14$ days, at which point burst-forming unit-erythroid (BFU-E) and colony-forming unit-granulocyte, monocyte (CFU-GM) colonies were counted using an inverted light microscope.

\section{RNA-seq and bioinformatics analysis}

Total RNA from isolated $\mathrm{CD} 4^{+}$cells (isolated using StemExpress) from both healthy and MDS BM specimens was obtained using the RNeasy Mini Kit (Qiagen). RNA was quantified in a NanoDrop 1000 and RNA quality was assessed by Agilent 2100 Bioanalyzer. Samples were then processed for RNA-Sequencing (RNA-seq) using the NuGen Ovation Human RNA-Seq Multiplex System (NuGEN Technologies). Briefly, $100 \mathrm{ng}$ of RNA was used to generate cDNA and a strand-specific library following the manufacturer's protocol. Quality control steps included BioAnalyzer library assessment and quantitative PCR for library quantification. The libraries were then sequenced using an Illumina NextSeq 500 v2 sequencer with 75-base pair (bp)-end runs to generate $\sim 60$ million reads per sample. Sequencing reads were subjected to adaptor trimming, quality assessment, and were aligned to human reference genome hs37d5 using Tophat v2.0.13 [20]. Quantification of aligned sequences associated with each gene was performed using HTSeq v0.6.1 [21] based on GENCODE release 19. Read counts of all samples were normalized using the median-of-ratios method implemented in R/Bioconductor package DESeq2 v1.6.3. Differential expression analysis between PD-1 and IgG control-treated samples was performed by serial dispersion estimation and statistical model-fitting procedures implemented in DESeq2 [22]. Genes with a $p$-value, adjusted for multiple testing with the Benjamini-Hochberg correction, of less than 0.05 were determined to be significantly differentially expressed. Gene set enrichment analysis 3.0 software was used to assess significant enrichment of biological pathways or gene sets in PD-1 versus Ig-treated patient and healthy donor datasets, respectively, against hallmark gene sets from the Molecular Signatures Database v6.2 [23].

\section{Statistics}

Data are presented as means \pm standard error of the mean. Differences between individual groups were analyzed by Student's $t$-test using Graphpad Prism version 7.03. $P$ values $<0.05$ were considered statistically significant. Significance was also confirmed with the Wilcoxon rank sum test. 


\section{Results}

\section{PD-1 and PD-L1 surface receptor expression is increased in MDS}

Given the unexplored role of the PD-1/PD-L1 pathway in MDS, and the poorly understood function of PD-1 in nonlymphoid cells, we first examined PD-1 surface receptor expression on HSPCs and erythroid progenitors isolated from the BM of MDS patients $(n=10)$ compared with normal donors $(n=6)$ (Supplementary Figure S2). All patients examined had significantly increased surface expression of PD-1 on both $\mathrm{CD} 71^{+}$erythroid progenitors $\left(P<0.05\right.$; Fig. 1a) and CD $34^{+}$HSPCs $(P<0.01$; Fig. 1b) versus the corresponding healthy donor $\mathrm{BM}$ populations, suggesting PD-1 upregulation may play a role in MDS. As PD-L1 ligation with PD-1 mediates immune suppression in the TME [24], we examined PD-L1 expression on $\mathrm{CD}_{3}{ }^{+} \mathrm{CD} 14^{+}$myeloid cells in $\mathrm{BM}$, which represent MDSCs. $\mathrm{CD} 33^{+} \mathrm{CD} 14^{+}$MDSCs from MDS BM showed marked upregulation of PD-L1 surface expression versus that on MDSC from the BM of normal donors $(P<0.005$; Fig. 1c). Finally, erythroid progenitors $(P<0.05$; Fig. 1d $)$ and HSPCs $(P<0.01$, Fig. 1e) from MDS patients also demonstrated significantly increased surface expression of PD-L1 versus expression in normal progenitors, indicating an autonomous regulation of immune checkpoint signaling in these MDS hematopoietic populations.

As PD-1/PD-L1 changes are most prominently studied in $\mathrm{T}$ cells in other tumor types, we also studied this population in MDS specimens. Total T cells, including both $\mathrm{CD}^{+}$and $\mathrm{CD}^{+} \mathrm{T}$ cells, were significantly reduced in primary MDS BM-MNC (Fig. 2a, c, and Supplementary Figure 3a; $P<$ $0.001, P<0.01$, and $P<0.01$, respectively), and the PD-1
Fig. 1 PD-1 and PD-L1 surface expression is increased in MDS. The percentage of PD- $1^{+}$and $\mathrm{PD}-\mathrm{L1}^{+}$hematopoietic cells was measured in BM-MNC isolated from MDS patients $(n=10)$ compared with healthy donors $(n=6)$ : a PD $-1^{+} \mathrm{CD} 71^{+}$ erythroid progenitors; $\mathbf{b}$ PD-1 ${ }^{+}$ CD $34^{+}$HSPCs; $\mathbf{c}$ PD-L1 ${ }^{+} \mathrm{CD} 33^{+}$ CD14 ${ }^{+}$MDSCs; d PD-L1 ${ }^{+}$ $\mathrm{CD} 71^{+}$erythroid progenitors; and e PD-L $1^{+} \mathrm{CD} 34^{+}$HSPCs.

Populations were gated on viable cells based on fluorescence minus one (FMO) controls (Supplementary Figure S2). $* P<0.05, * * P<$ $0.01, * * * P<0.005$; data are presented as mean \pm standard error of the mean
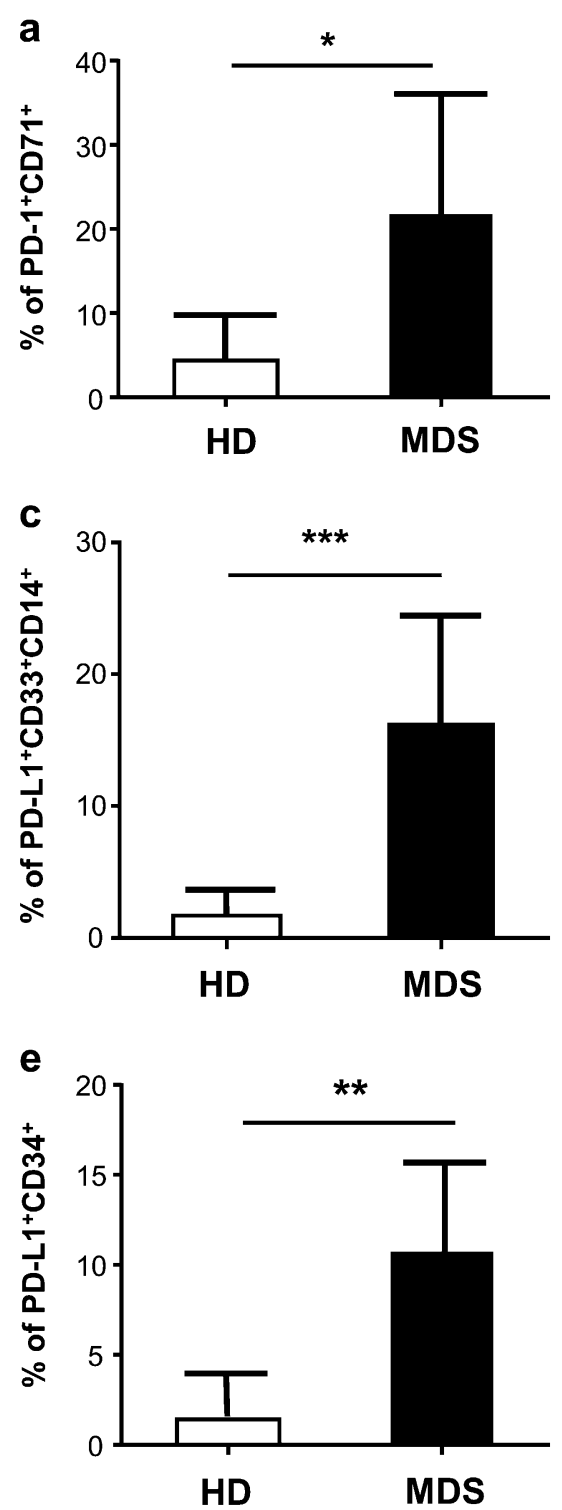
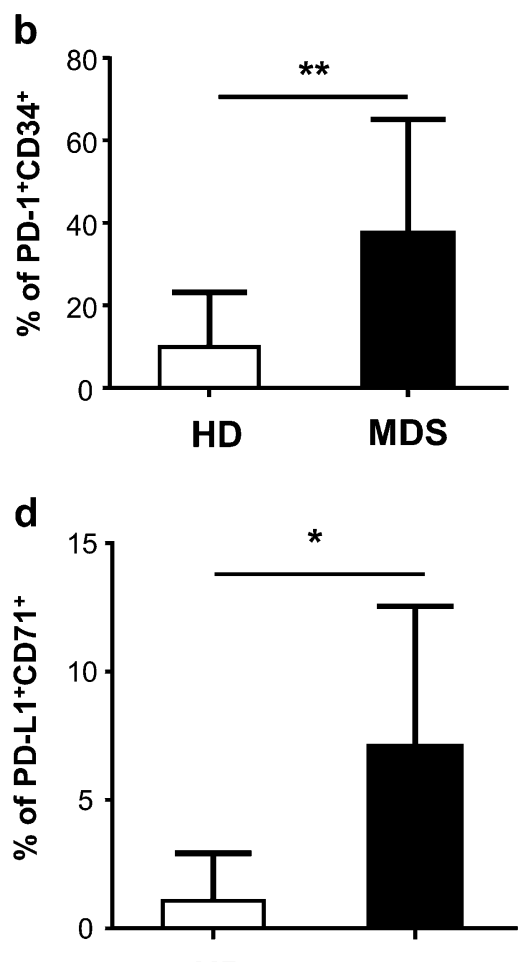

HD

MDS 
Fig. 2 PD-1 expression is increased in MDS patient T cells present in the bone marrow niche. The percentage of $\mathrm{CD} 4^{+}$ and $\mathrm{CD} 8^{+} \mathrm{T}$ cells and their expression of PD-1 were measured in BM-MNC isolated from MDS patients $(n=10)$ compared with healthy donors $(n=6)$ : a percent $\mathrm{CD}^{+}{ }^{+} \mathrm{T}$ cells; b percent $\mathrm{CD} 4{ }^{+} \mathrm{PD}-1^{+}$cells; $\mathbf{c}$ percent $\mathrm{CD}^{+} \mathrm{T}$ cells; and $\mathbf{d}$ percent $\mathrm{CD} 8{ }^{+} \mathrm{PD}-1^{+}$cells. Populations were gated on viable cells based on fluorescence minus one (FMO) controls. $* * P<0.01, * * * P<$ $0.005, * * * * P<0.001$; data are presented as mean \pm standard error of the mean
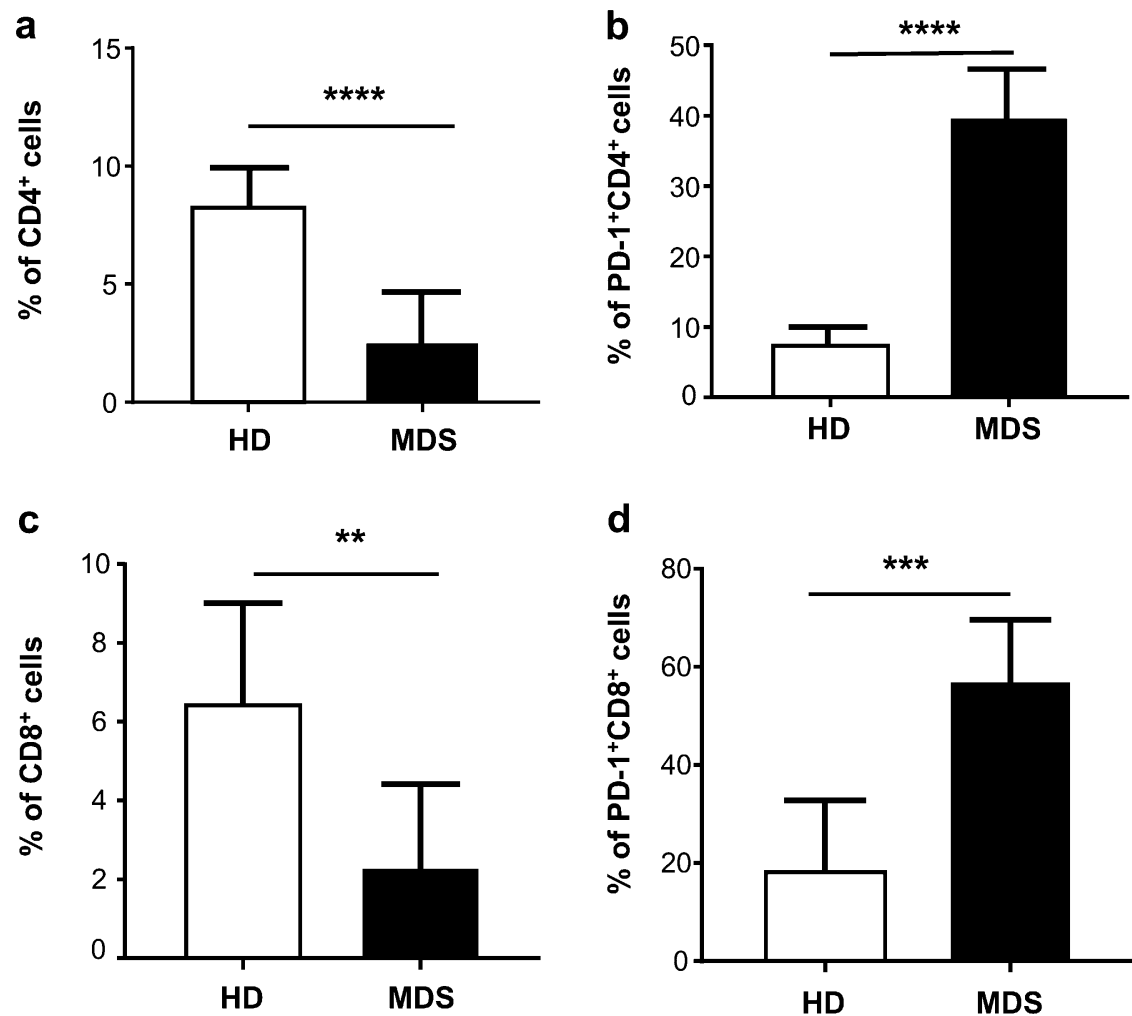

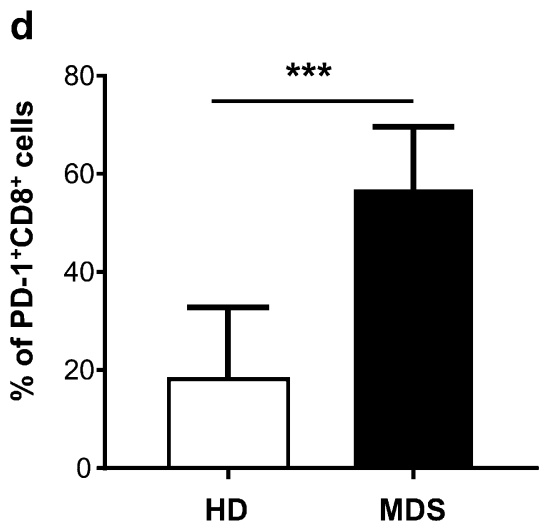

expression was increased in these cells versus $\mathrm{CD}^{+}$and $\mathrm{CD}^{+} \mathrm{T}$ cells from normal BM-MNC (Fig. 2b, d; $P<0.001$ and $P<0.005$, respectively). Finally, there was an upregulation of Tregs in MDS BM-MNC compared to healthy controls (Supplementary Figure 3b, $P<0.01$ ). We conclude that the MDS BM niche is immune suppressive.

\section{PD-1 and PD-L1 are overexpressed in aged S100A9Tg mice}

Akin to human MDS, S100A9Tg mice, where S100A9 expression is driven by the $\mathrm{H} 2 \mathrm{~K}$ (myeloid) promoter, display age-dependent activation of MDSC that results in hematopoietic progenitor cell death and dysplastic, ineffective hematopoiesis [9]. Using this mouse model, we assessed the expression of PD-1 and PD-L1 in the BMMNC and MDSC of young and old S100A9Tg mice (3-4 and 14-16 months old, respectively) compared with agematched wild-type (WT) littermates (Supplementary Figure S4). c-Kit ${ }^{+} \mathrm{Lin}^{-} \mathrm{CD} 16{ }^{-} \mathrm{CD} 32^{-}$common myeloid progenitor (CMP) cells derived from BM of young WT and S100A9Tg mice showed little expression of PD-1 and PDL1 expression on (Fig. 3a, b). In contrast, PD-1 and PD-L1 expression levels on CMPs were significantly greater in older versus younger mice, and there were marked increases in PD-1 (Fig. 3a, $P<0.005$ ) and PD-L1 (Fig. 3b, $P<0.05$ ) expression in aged S100A9Tg mice versus aged WT littermates; thus, S100A9 induces PD-1 and PD-L1 surface expression beyond that which is manifest with normal aging. Notably, increased PD-L1 surface receptor expression was also observed on $\mathrm{Gr}-1^{+} \mathrm{CD} 11 \mathrm{~b}^{-}$cells that represent MDSCs in S100A9Tg mice (Fig. 3c). Thus, forced expression of S100A9 is sufficient to augment an immune suppression PD-L1/PD-1 circuit driven by MDSC.

Given $\mathrm{T}$ cell deficits in the BM niche of MDS patients, we also assessed T-cell numbers in S100A9Tg mice. There was no statistically significant difference in the numbers of $\mathrm{CD}^{+}{ }^{+}$or $\mathrm{CD}^{+}$cells in WT versus $\mathrm{S} 100 \mathrm{~A} 9 \mathrm{Tg}$ littermates, although increased PD-1 expression was observed in the $\mathrm{CD}^{+}$cells of S100A9Tg mice versus WT littermates (Supplementary Figure 4d). Comparing the difference in $\mathrm{T}$ cell PD-1 expression between human and mice suggests the PD-1/PD-L1 axis changes in T cells may be independent of its effects on HSPC and hence their function may be more in immunesurveillance rather than hematopoietic suppression.

\section{S100A9 directly induces PD-1 expression on progenitors and PD-L1 on MDSC}

To test if S100A9 can directly induce PD-1 and PD-L1 cell surface expression in primary hematopoietic cells, BMMNC isolated from healthy donors $(n=5)$ were treated with $10 \mu \mathrm{g} / \mathrm{mL}$ recombinant human S100A9 (rhS100A9) or IgG (control) for $48 \mathrm{~h}$ (Supplementary Figure S5). Treatment with rhS100A9 led to significant increases in 


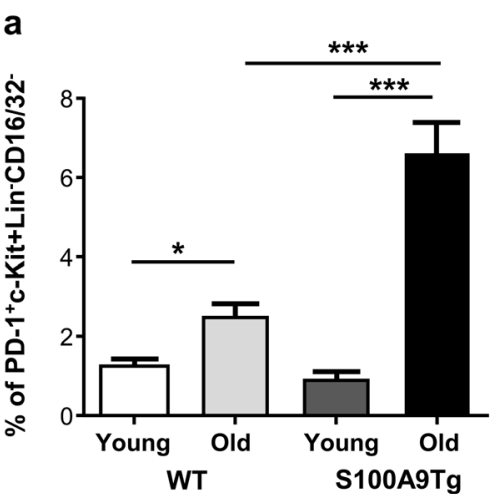

Fig. 3 PD-1 and PD-L1 expression levels are elevated in the CMP and MDSC of S100A9Tg mice. The percentage of PD- $1^{+}$and PD-L1 ${ }^{+}$ hematopoietic cells was measured in BM isolated from age-matched (young, 3-4 months; old, 14-16 months) S100A9Tg and WT littermates. Five mice were used per group and the experiment was repeated

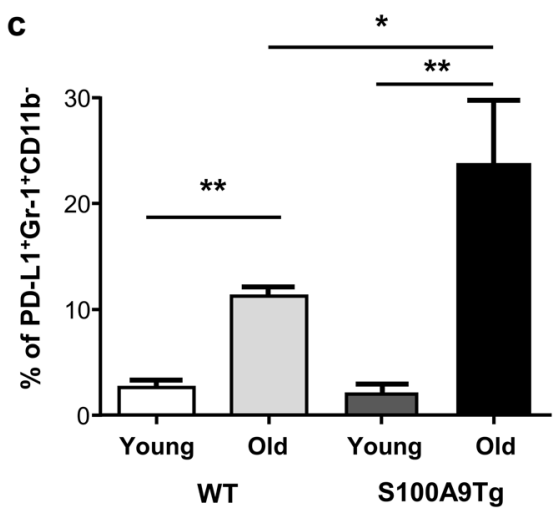

three times. a PD- ${ }^{+} \mathrm{c}-\mathrm{Kit}^{+} \mathrm{Lin}^{-} \mathrm{CD} 16 / 32^{-} \mathrm{CMP}$ cells; b PD-L1 ${ }^{+} \mathrm{CMP}$ cells; and $\mathbf{c ~} \mathrm{Gr}-1^{+} \mathrm{CD} 11 \mathrm{~b}^{-}$MDSCs. Populations were gated on viable cells based on fluorescence minus one (FMO) controls (Supplementary Figure S3). $* P<0.05, * * P<0.01, * * * P<0.005$; data are presented as mean \pm standard error of the mean
PD-L1 expression on $\mathrm{CD}_{3}{ }^{+} \mathrm{CD} 14^{+}$MDSCs in all healthy donors analyzed (Fig. $4 \mathrm{a} ; P<0.01$ ). Furthermore, rhS100A9 treatment also led to significant increases in PD-1 cell surface expression on both CD34 ${ }^{+}$HSPCs (Fig. 4b; $P<0.01$ ) and $\mathrm{CD}^{+} 1^{+}$erythroid progenitors (Fig. 4c; $P<0.01)$.

BM plasma concentrations of S100A9 are significantly elevated in MDS patients compared with healthy donors $[9,10]$. To test if BM plasma-derived S100A9 can induce surface receptor expression of PD-1 and PD-L1, healthy donor BM-MNC were incubated with each MDS patient plasma (patients 1, 3, 7, 12, and 15 from Supplementary Table 1). In accordance with the effects of rhS100A9 treatment, incubation of healthy donor BM-MNC with MDS patient plasma triggered significant increases in the percentage of PD $-1^{+} \mathrm{CD} 34^{+}$HSPCs (Fig. 4d; $P<0.05$ ) and PD- ${ }^{+}{ }^{C D} 71^{+}$erythroid progenitors (Fig. 4e; $P<0.005$ ). Thus, S100A9 directly induces the expression of PD-1 on HSPCs and its corresponding ligand PD-L1 on MDSC.

\section{The S100A9 to PD-1/PD-L1 axis induces hematopoietic cell death}

PD-1 and PD-L1 engagement induces T-cell exhaustion and apoptosis [12, 13]. Thus, we tested if PD-1 engagement on HSPCs with PD-L1 induces cell death and contributes to the ineffective hematopoiesis that is a hallmark of MDS. BMMNC isolated from healthy donors $(n=5)$ were treated with $10 \mu \mathrm{g} / \mathrm{mL}$ rhS100A9 or IgG (control) for $48 \mathrm{~h}$, and cells were then assessed for levels of surface PD-1 and PDL1 and anti-active caspase- 3 expression, a marker of cell death. The percentage of CD34 ${ }^{+}$HSPCs and CD71 ${ }^{+}$erythroid progenitors with active caspase-3 was significantly increased by rhS100A9 treatment (Fig. 5a, b; $P<0.005$ ). To assess if engagement of PD-1 was also sufficient to provoke hematopoietic progenitor cell death and if this was augmented in MDS, BM-MNC isolated from MDS patients $(n=3)$ and healthy donors $(n=3)$ were cultured in plates coated with or without recombinant human PD-L1 for $24 \mathrm{~h}$ and levels of activated caspase- 3 were determined by flow cytometry. PD-1/PD-L1 ligation resulted in marked increases in activated caspase- 3 in $\mathrm{CD}^{+} 4^{+}$HSPCs and $\mathrm{CD}_{71}{ }^{+}$erythroid progenitors from MDS patients but only slightly in normal donors (Fig. 5c, d). Thus, PD-1/PD-L1 interactions may contribute to progenitor cell death that is manifest in MDS.

\section{PD-1/PD-L1 pathway blockade promotes effective hematopoiesis in MDS}

To test if blocking PD-1/PD-L1 interaction could improve hematopoiesis in MDS, colony-forming capacity was assessed after treating MDS BM-MNC $(n=5)$ with antiPD-1 or anti-PD-L1 blocking antibody for $48 \mathrm{~h}$. Notably, PD-1 and PD-L1 blockade significantly improved CFU-GM $(P<0.01$ and $P<0.05$, respectively) and BFU-E $(P<0.05$ for both) colony-forming capacity versus IgG controltreated BM-MNC (Fig. 6a), suggesting PD-1/PD-L1 blockade may be a beneficial treatment option to promote effective hematopoiesis in MDS. To assess if hematopoietic improvement with PD-1 blockade is also manifest in vivo, aged S100A9Tg $(n=6)$ and WT littermates $(n=6)$ were treated with $150 \mu \mathrm{g} / \mathrm{mouse}$ anti-PD-1 blocking antibody twice a week for 6 weeks, and complete blood counts were measured weekly. Anti-PD-1 treatment of S100A9Tg mice significantly increased red blood cell (RBC) and white blood cell (WBC) counts versus IgG controls (Fig. 6b). In contrast, anti-PD-1 treatment of WT mice had no effect on $\mathrm{RBC}$ and WBC counts, as expected given that WT BM progenitors express low levels of PD-1 and PD-L1 (Fig. 3). 
Fig. 4 S100A9 induces the expression of PD-1 on progenitors and PD-L1 on MDSCs. The percentage of PD$1^{+}$and PD-L1 ${ }^{+}$hematopoietic cells was measured in BM-MNC isolated from healthy donors $(n=5)$ treated with $10 \mu \mathrm{g} / \mathrm{mL}$ rhS100A9 or control IgG (control) (a-c), or with MDS patient or healthy donor BM plasma (d, e) for $48 \mathrm{~h}$. a PD-L1 ${ }^{+} \mathrm{CD} 33{ }^{+} \mathrm{CD} 14^{+} \mathrm{MDSC}$; b PD-1 ${ }^{+} \mathrm{CD} 34^{+}$HSPC; c PD- $1^{+} \mathrm{CD} 71^{+}$ erythroid progenitors; $\mathbf{d}$ PD-1 ${ }^{+} \mathrm{CD} 34^{+}$HSPCs; and e PD-1 ${ }^{+} \mathrm{CD} 71^{+}$erythroid progenitors. Populations were gated on viable cells based on fluorescence minus one (FMO) controls (Supplementary Figure S4). $* P<0.05, * * P<$ 0.01 ; data are presented as mean \pm standard error of the mean
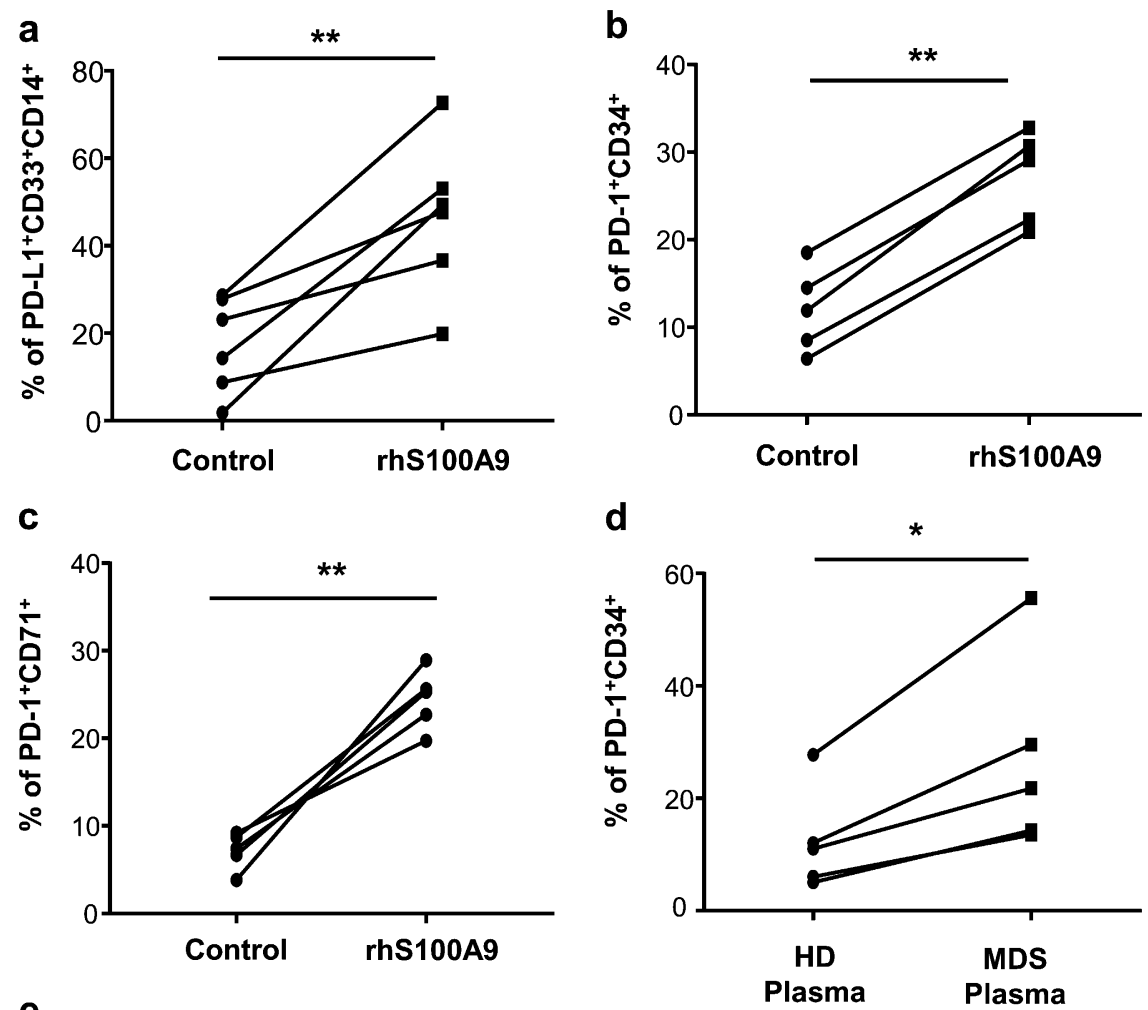

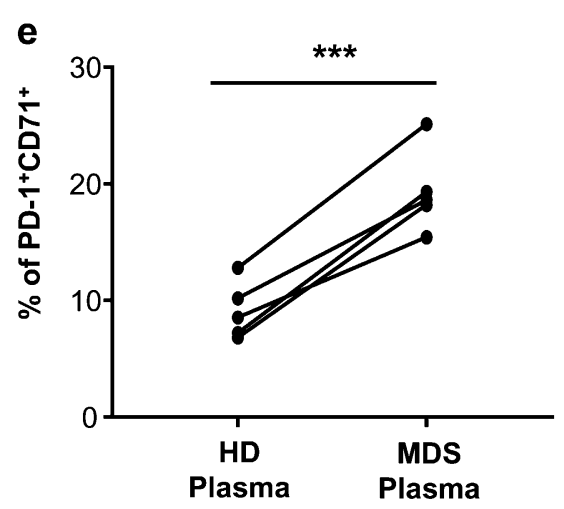

To test if increased complete blood counts in S100A9Tg mice reflected improved hematopoiesis, BM cells from the anti-PD-1-treated S100A9Tg and WT mice were isolated to assess colony-forming capacity. As expected, BFU-E and CFU-GM colony-forming capacity was significantly reduced in S100A9Tg versus WT mice treated with control IgG $(P<0.05$; Fig. 6c). Importantly, anti-PD-1-treated S100A9Tg mice had significant increases in BFU-E colony-forming capacity versus WT-treated mice (Fig. $6 \mathrm{c}, P<$ $0.01)$. There were also modest increases of CFU-GM in anti-PD-1-treated S100A9Tg mice. Similarly, BFU-E and CFU-GM in S100A9Tg BM were significantly increased with anti-PD-L1 treatment (Supplementary Figure S6). These data suggest that PD-1 and PD-L1 blockade is an attractive therapeutic strategy to restore effective hematopoiesis in MDS patients.

\section{S100A9-induced expression of PD-1 and PD-L1 is} associated with abnormal c-Myc activation

Recent studies have suggested that abnormal metabolic changes driven by the c-Myc (MYC) oncogenic transcription factor during tumorigenesis lead to PD-1/PD-L1 pathway activation and immunosuppression [11, 25]. Aged S100A9Tg mice display robust activation of S100A9induced inflammatory pathways that lead to abnormal metabolic changes, as evidenced by increased body weight, insulin resistance, and hyperglycemia [26]. To assess the possible links of S100A9/PD-1/PD-L1 pathway activation and MYC in MDS, MYC protein levels were compared in BM-MNC from MDS patients versus healthy donors. Consistently ( $n=5$ total), PD-1, PD-L1, and MYC protein expression were elevated in MDS BM-MNC versus healthy 
Fig. 5 S100A9/PD-1/PD-L1 axis induces hematopoietic cell death. a, $\mathbf{b}$ The percentage of active caspase- $3^{+}$hematopoietic cells was measured in BM-MNC isolated from healthy donors $(n=5)$ treated with $10 \mu \mathrm{g} / \mathrm{mL}$ rhS100A9 or control IgG (control) for $48 \mathrm{~h}$. Populations were gated on viable cells based on fluorescence minus one (FMO) controls: percentage of active caspase- 3 a CD34+ HSPCs and b CD71+ erythroid progenitors. $* * * P<0.001$; data are presented as mean \pm standard error of the mean. $\mathbf{c}, \mathbf{d}$ Two million BM-MNC were plated per well in 24-well plates coated with recombinant human PD-L1 $(2 \mu \mathrm{g} / \mathrm{mL})$ for $24 \mathrm{~h}$ at $4{ }^{\circ} \mathrm{C}$. The percentage of active caspase- $3^{+} \mathbf{c}$ CD $34^{+}$HSPCs and d CD71 ${ }^{+}$ erythroid progenitors are shown in a representative sample from three healthy donors and MDS patients a

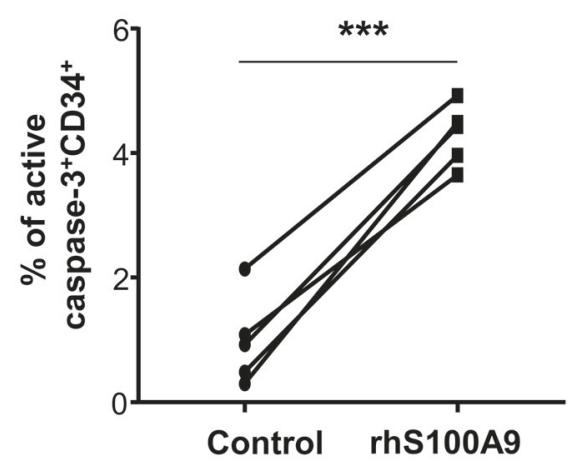

b

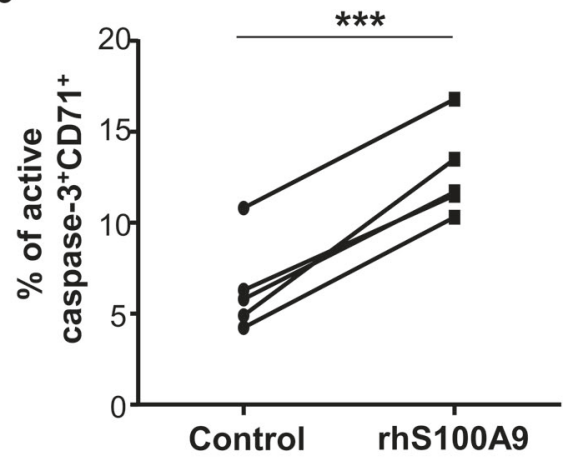

C

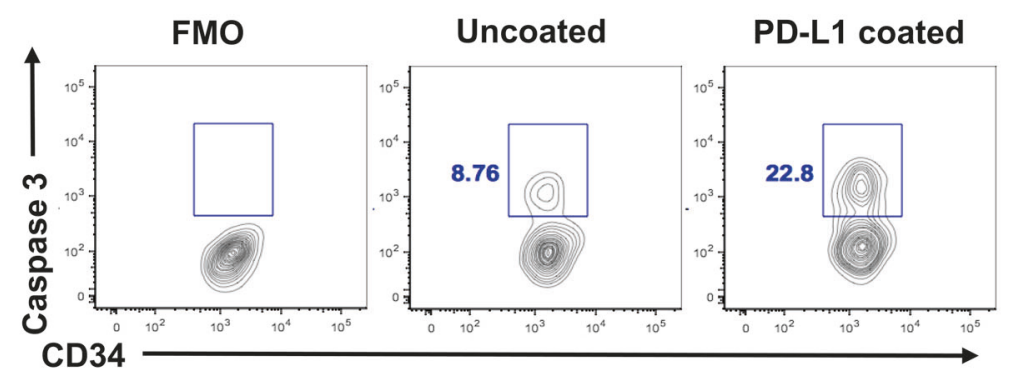

d

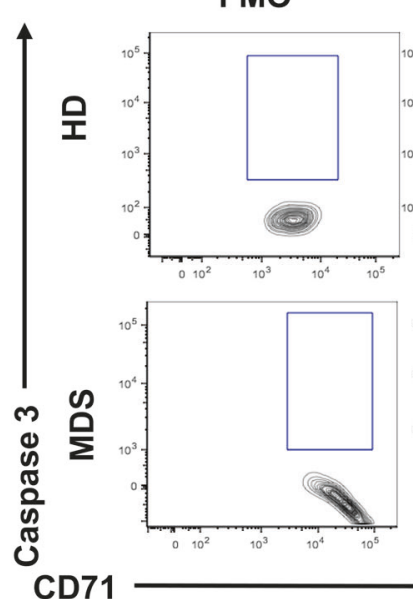

Uncoated

PD-L1 coated
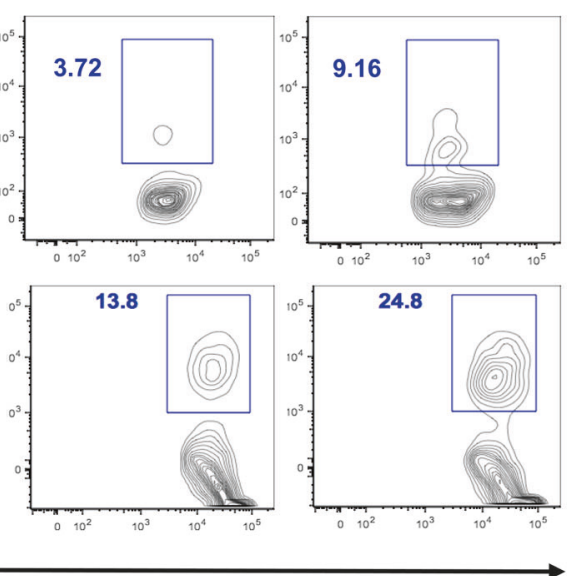

donors (Fig. 7a). Further, the treatment of healthy donor BM-MNC with $10 \mu \mathrm{g} / \mathrm{mL}$ rhS100A9 for $48 \mathrm{~h}$ was sufficient to induce the expression of PD-1, PD-L1, and MYC versus untreated cells (Fig. 7b). Conversely, RNA-seq analyses of MDS HSPC treated or not treated with anti-PD-1 for $48 \mathrm{~h}$ revealed a decrease in MYC target genes following treatment with anti-PD-1, along with increases in the expression of genes involved in heme metabolism and IL2/ STAT5 signaling, as would be expected with improvements in hematopoiesis (Fig. 7c, and Supplementary Figure S7a).

To test if Myc contributes to S100A9-directed increases in the cell surface expression of PD-1 and PD-L1 on BMMNC, we assessed the effects of Myc heterozygosity using BM from heterozygous $M y c^{+/-}$mice and WT littermates; $M y c^{+/-}$BM cells expressed reduced levels of Myc protein versus WT littermate BM cells as judged by immunoblots (Supplementary Figure S7b). These cells were then treated with increasing concentrations of S100A9 for $48 \mathrm{~h}$ and PD-1 and PD-L1 cell surface expression was assessed on c-Kit ${ }^{+} \mathrm{Lin}^{-} \mathrm{CD} 16 / 32^{-}$CMP cells by flow cytometry. The surface receptor expression of PD-1 (Fig. 7d top panel) and PD-L1 (Fig. 7e top panel) increased in an S100A9 concentration-dependent manner on WT-derived BM cells. Conversely, S100A9 treatment was insufficient to induce PD-1 and PD-L1 cell surface receptor expression in heterozygous $\mathrm{Myc}^{+/-}$BM cells (Fig. 7d, e bottom panels). Thus, Myc contributes to the control of the PD-1/PD-L1 axis by S100A9.

To gain insights into the mechanism by which S100A9 controls the expression of PD-1, PD-L1, and Myc, 
Fig. 6 PD-1/PD-L1 pathway blockade augments the colonyforming capacity of MDS patient BM progenitors and improves hematopoiesis in S100A9Tg mice. a MDS BMMNC $(n=5)$ were treated with anti-PD-1 or anti-PD-L1 blocking antibody for $48 \mathrm{~h}$, and cells were then plated to assess CFU-GM and BFU-E colonyforming capacity versus cells treated with control IgG. b, c Aged (14 months) S100A9Tg $(n=6)$ and agematched WT mice $(n=6)$ were treated with $150 \mu \mathrm{g} / \mathrm{mouse}$ antiPD-1 blocking antibody twice a week for 6 weeks. b Complete blood counts were measured weekly, and mean RBC and WBC counts are shown through week 6. c BM cells from the aged, anti-PD-1-treated S100A9Tg and WT mice were isolated and BFU-E and CFUGM colony-forming capacity was determined. $* P<0.05$, $* * P<0.01$; data are presented as mean \pm standard error of the mean. BFU-E burst forming unit-erythroid; CFU-GM colony-forming unitgranulocyte, monocyte; RBC red blood cell; WBC white blood cell
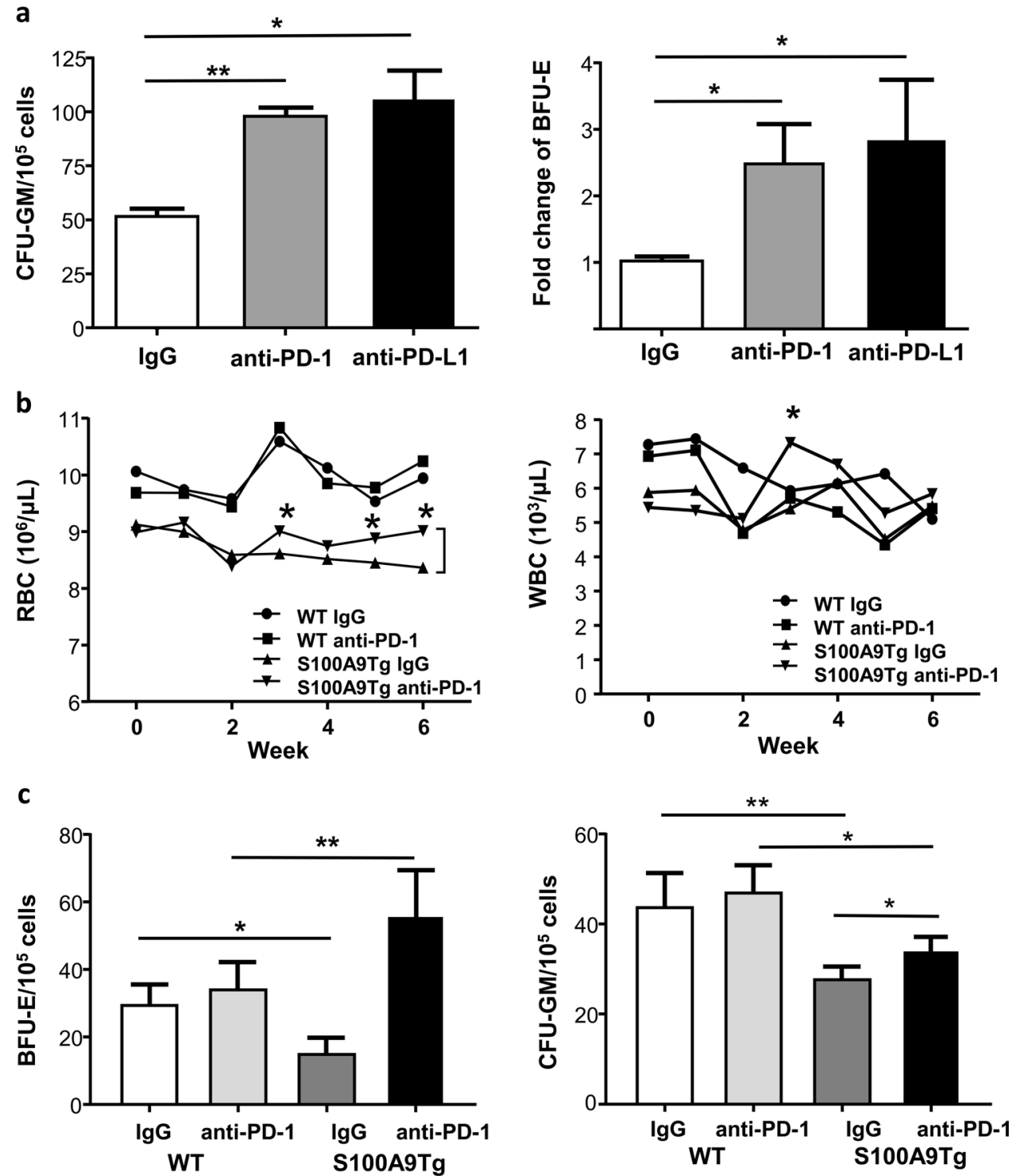

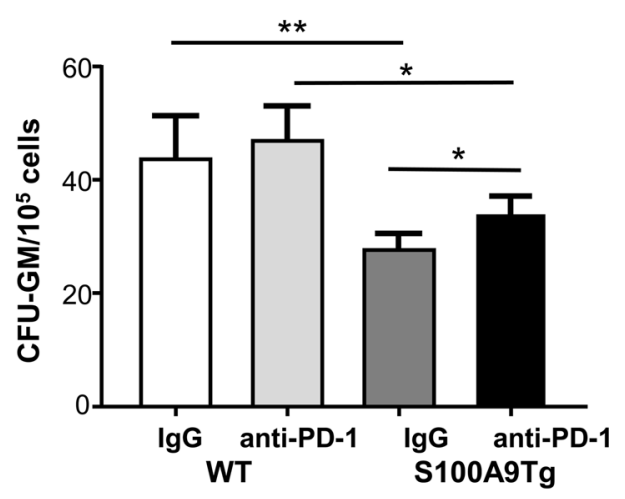

quantitative real-time PCR analyses were performed. These studies revealed that $P D-1, P D-L 1$, and $M y c$ mRNA levels are elevated in S100A9Tg versus WT BM-MNC (Fig. 8a) and in MDS patient versus normal BM-MNC (Fig. 8b). Collectively these findings suggest that S100A9 induction of Myc triggers increases in PD-1 and PD-L1 expression that activate MDSC, provoke HSPC cell death, and lead to immune evasion.

\section{Discussion}

Despite recent advances in our understanding of MDS pathobiology, reversing ineffective hematopoiesis remains a major therapeutic challenge, given that the precise signaling pathways mediating HSPC defects are not fully understood. Our recent investigations have shown that a chronic inflammatory response, coupled with expansion of hematopoietic-inhibitory MDSCs, directs HSPC injury and clonal selection in MDS [9, 10, 27]. Within the BM niche, MDSCs serve as a paracrine source of the alarmin S100A9, which activates and expands MDSCs, and is sufficient to provoke cell death of HSPCs to drive ineffective hematopoiesis $[9,10]$. These findings have been validated by others in alternate models, namely deletion $5 \mathrm{q}(\operatorname{del}[5 \mathrm{q}])$ MDS and the Rps14-haplodeficient murine model, whereby S100A9 and S100A8 (calprotectin), the heterodimeric binding partner of S100A9, trigger erythroid progenitor cell death [28].

An emerging concept is that the local BM microenvironment in MDS is also abnormal and plays critical roles in MDS pathobiology. Here, aging-associated inflammation, or "inflammaging," likely contributes to genetic instability and a suppressive BM microenvironment, 
a

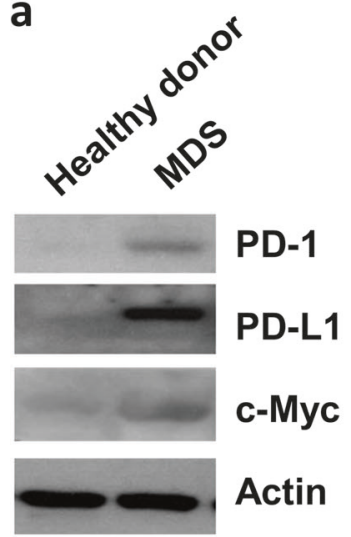

b

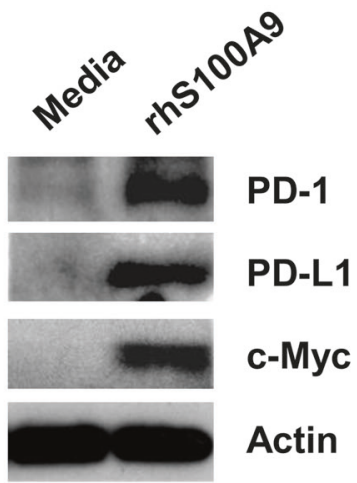

C

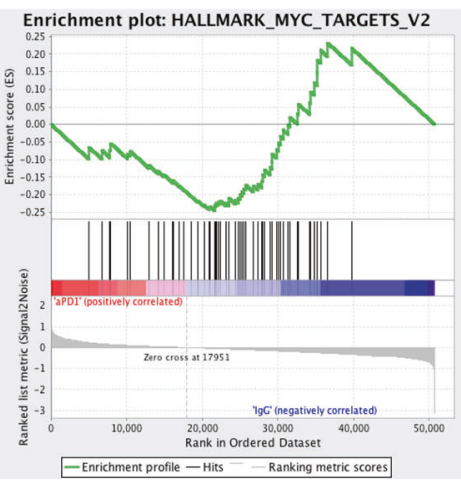

d

rhS100A9

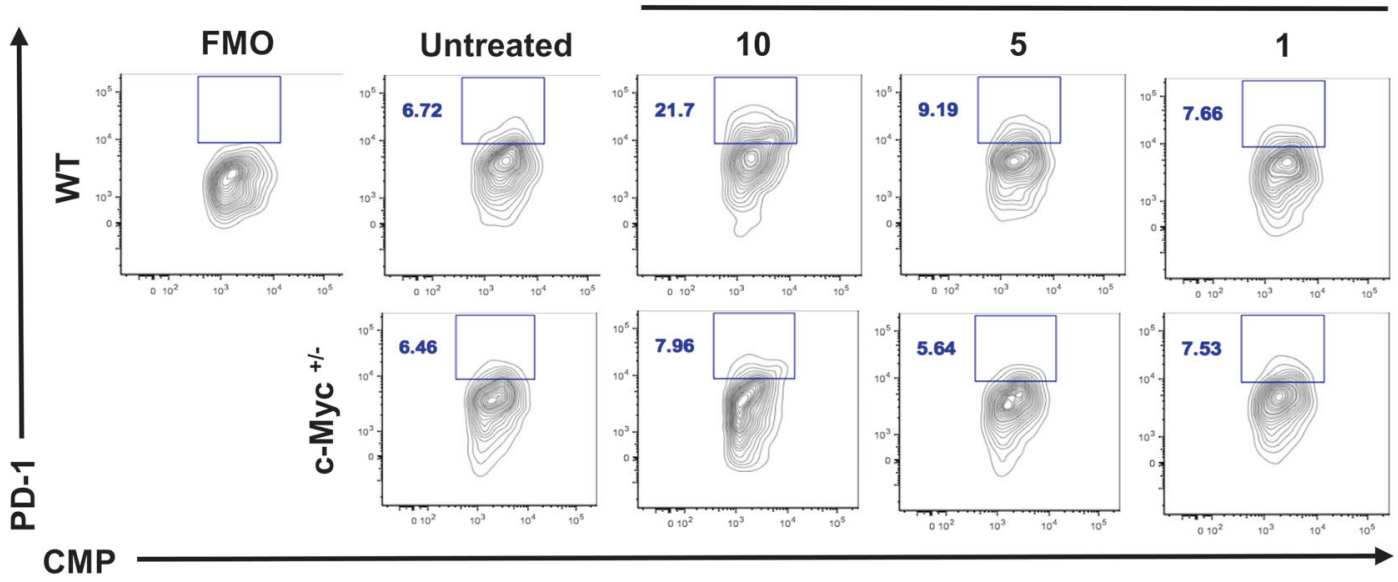

e

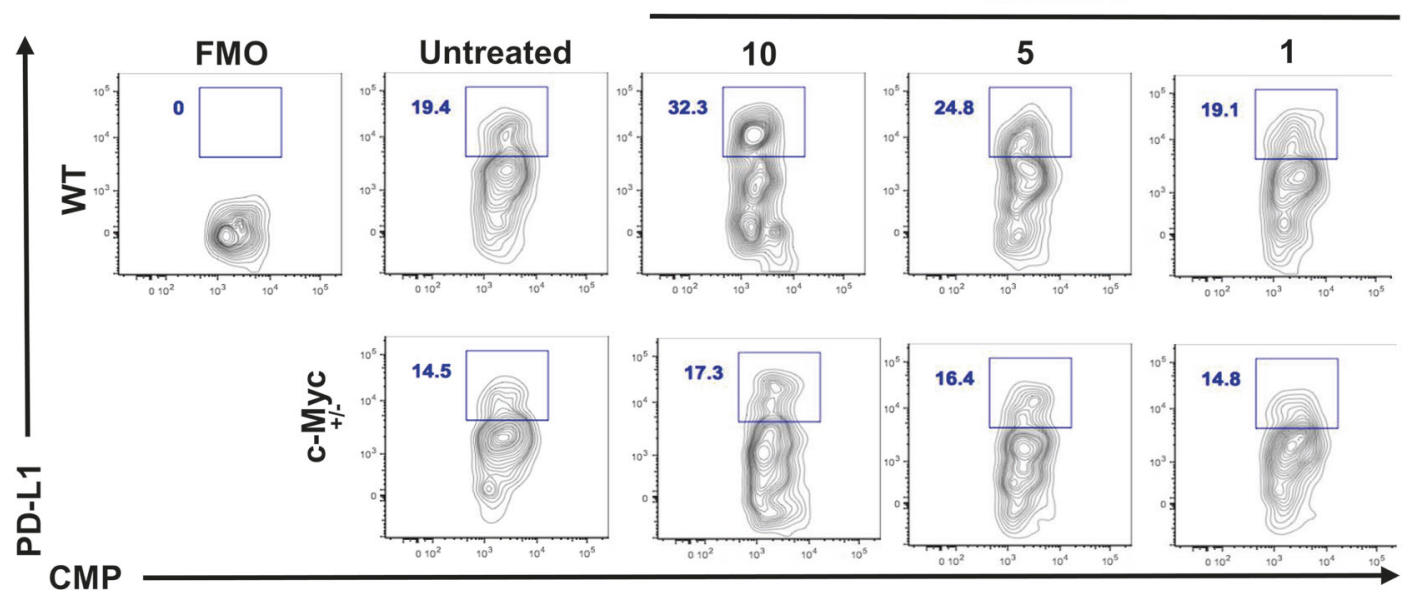

Fig. 7 S100A9/PD-1/PD-L1 pathway activation augments MYC expression. Western blot was performed to assess levels of PD-1, PD$\mathrm{L1}$, and MYC protein in a MDS versus healthy donor BM-MNC and $\mathbf{b}$ healthy donor BM-MNC treated with $10 \mu \mathrm{g} / \mathrm{mL}$ rhS100A9 for $48 \mathrm{~h}$. Data are representative of five independent experiments. (c) RNA-seqbased transcriptional network analysis indicating downregulation of Hallmark Myc pathways in anti-PD1-treated CD34+ HSPC $(n=5)$.
The percentage of $\mathrm{PD}-1^{+}$and $\mathrm{PD}-\mathrm{L}^{+}{ }^{+} \mathrm{c}-\mathrm{Kit}^{+} \mathrm{Lin}^{-} \mathrm{CD} 16 / 32^{-} \mathrm{CMP}$ cells was measured in BM cells isolated from $\mathrm{Myc}^{+l-}$ and WT littermates and treated with increasing concentrations of S100A9. Populations were gated on viable cells based on fluorescence minus one (FMO) controls. The percentage of $\mathbf{d}$ PD $-1^{+}$and e PD-L1 ${ }^{+}$CMP cells in the BM of representative samples from five $\mathrm{Myc}^{+/-}$and WT mice is shown 


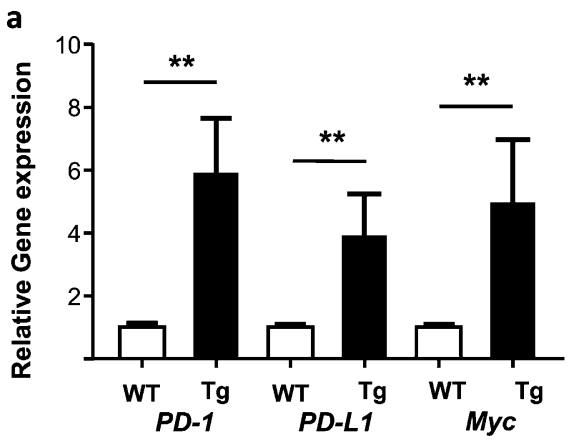

Fig. $8 M Y C, P D-1$, and $P D-L 1$ expression levels are elevated in the BM-MNC of MDS patients and of S100A9Tg mice. a qRT-PCR analysis of BM-MNC isolated from WT $(n=5)$ and S100A9Tg $(n=$ $5)$ aged littermate mice were analyzed for the expression of $P D-1, P D$ -

and to defective HSPCs [9, 10, 29-35]. Indeed, S100A9 expression is also induced in mesenchymal stromal cells, where it contributes to DNA damage in HSPCs via reactive oxygen species and p53-dependent pathways [36]. Importantly, S100A9 is also implicated in clonal expansion and leukemia progression in both primary MDS specimens and in the S100A9Tg mouse model that phenocopies human MDS [36]. Finally, S100A9 can also suppress the transcription and cellular elaboration of erythropoietin [37]. Collectively, these findings establish central roles for S100A9 in MDS pathobiology, whereby aberrant S100A9driven signaling results in pathobiological consequences in the entire BM niche.

To prevent unwarranted damage and maintain homeostasis, normal proinflammatory responses include events that suppress inflammation and dampen the immune response. One such mechanism of the immune system is governed by the immune checkpoint receptor PD-1 and its ligand PD-L1, where engagement of PD-1 induces T-cell exhaustion and apoptosis [12, 13]. Strikingly, here our studies revealed nontraditional roles (i.e., non-T-cell-specific) for PD-1 and PD-L1 in MDS, where S100A9 contributes to ineffective hematopoiesis in MDS via induction of PD-1 and PD-L1 expression. Importantly, checkpoint inhibitor treatment both in vitro and in vivo is shown to improve hematopoiesis in MDS, supporting the notion that anti-PD-1 or anti-PD-L1 alone or in combination with other therapies will show a benefit for MDS patients.

MDS patients express increased levels of PD- 1 and PD$\mathrm{L} 1$ on $\mathrm{CD} 34^{+} \mathrm{HSPCs}$ and $\mathrm{CD} 71^{+}$erythroid progenitors versus age-matched healthy donors, whereas $\mathrm{CD} 33^{+} \mathrm{CD} 14^{+}$ MDSC express elevated levels of PD-L1 in MDS. Similar increases in PD-1 and PD-L1 expression are manifest in aged S100A9Tg mice and in healthy donor BM-MNC treated with rhS100A9; thus, S100A9 is sufficient to provoke increases in PD-1 and PD-L1 expression. Further, PD$\mathrm{L} 1$ can directly induce $\mathrm{CD} 34^{+} \mathrm{HSPC}$ and $\mathrm{CD} 71^{+}$erythroid

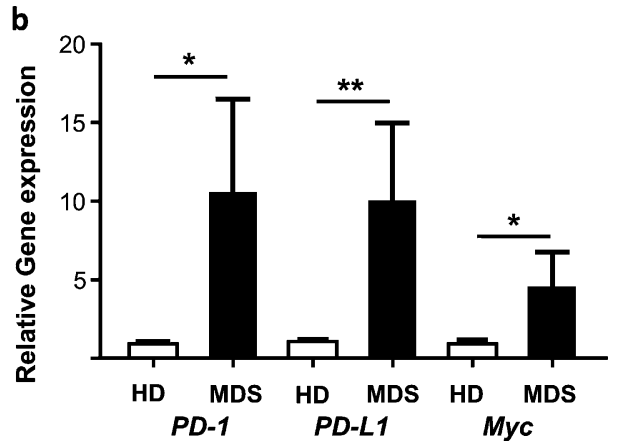

$L 1$, and Myc. b qRT-PCR analysis of BM-MNC isolated from healthy $(n=5)$ versus MDS $(n=5)$ patients. $* P<0.05, * * P<0.01, * * * P<$ 0.005 ; data are presented as mean \pm standard error of the mean

progenitor cell death, suggesting PD-1/PD-L1 ligation contributes to ineffective hematopoiesis in MDS. Consistent with this notion, PD-1 or PD-L1 blockade markedly improves the hematopoietic colony-forming capacity of MDS BM-MNC. Similarly, in vivo treatment of S100A9Tg mice with PD-1 or PD-L1 blocking antibody significantly improves hematopoiesis, as evidenced by improved complete blood cell counts and increased colony-forming capacity. Collectively, these data highlight the therapeutic potential of PD-1/PD-L1 pathway blockade in MDS.

Notably, Myc is known to induce the transcription of PD-L1 [11], and our studies have shown that Myc contributes to S100A9-mediated control of PD-1/PDL1 surface receptor expression. Interestingly, Myc is a major regulator of tumor cell metabolism, and here we have found that BM-MNC of MDS patients and healthy BMMNC treated with rhS100A9 express elevated Myc levels, and that MDS patients and S100A9Tg mice display features of metabolic syndrome [38], including hyperglycemia in the BM niche. We posit that high concentrations of S100A9 produced by MDSCs in the BM niche, as well as other inflammatory cytokines such as IL-10 and TGF- $\beta$, provoke an abnormal metabolic profile via activation of Myc. While the mechanisms of Myc activation downstream of S100A9 will need to be elucidated, our data have previously demonstrated the activation of $\beta$-catenin which has as its downstream targets Myc [10, 39]. Regardless of the precise wiring, S100A9 control of PD-1/PD-L1 is shown to clearly involve Myc, which is known to induce $P D-L 1$ transcription in tumors to facilitate immune evasion [25], as $M y c$ heterozygosity significantly dampens the expression of these checkpoints.

S100A9-directed control of the PD-1/PD-L1 axis also has clinical implications. First, S100A9 expression appears unaffected by the epigenetic drug 5-azacytidine [40], and S100A9-directed induction of PD-1/PD-L1 may contribute to therapeutic resistance of MDS to 5-azacytidine, which 
also induces the expression of these immune checkpoints [15]. Second, our RNA-seq analysis of primary human MDS HSPC treated with anti-PD-1 antibody suggests the relevance of the S100A9-PD-1/PD-L1 circuit would also affect the use of erythropoietin as a hematopoietic stimulating agent in combination with anti-PD-1/PD-L1 treatment. Specifically, anti-PD-1 treatment induces the expression of the heme metabolic pathway genes as well as the Epo/STAT5 pathway, which are known regulators of erythroid cell proliferation and survival in MDS [41, 42]. This suggests that the improved BFU-E colony formation of MDS BM after treatment with anti-PD-1/PD-L1 in our studies involves Epo/STAT5 regulation, which will be an important aspect of future analyses.

Collectively, these findings (1) expand our understanding of the role of PD-1/PD-L1 interaction beyond T cells, which has implications for general immunology and immunotherapy; (2) demonstrate that S100A9, which is instrumental in the pathogenesis of MDS, plays a critical role in the induction of PD-1/PD-L1 surface receptor expression on HSPCs and MDSCs; and (3) suggest that PD-1/PDL1 signaling contributes to ineffective hematopoiesis in MDS. Finally, and importantly, these data suggest that antiPD-1 or anti-PD-L1 blocking antibodies, alone or in combination with other strategies, offer therapeutic promise in MDS to improve the BM microenvironment and restore effective hematopoiesis.

Acknowledgements This work was supported by the Department of Defense Bone Marrow Failure Research Program grant W81XWH-151-0440 (SW), by a James \& Esther King Discovery Science Award from the State of Florida (SW), by National Institutes of Health K01 career award CA187020-02 (EAE), T32 training grant CA115308 (NT), by the Cortner-Couch Endowed Chair for Cancer Research from the University of South Florida School of Medicine (JLC), by funds from an LLS-SCOR award (to SW, AFL, and JLC), and by the National Cancer Institute Cancer Center Support Grant P30CA076292.

\section{Compliance with ethical standards}

Conflict of interest The authors declare that they have no conflict of interest.

Publisher's note: Springer Nature remains neutral with regard to jurisdictional claims in published maps and institutional affiliations.

Open Access This article is licensed under a Creative Commons Attribution 4.0 International License, which permits use, sharing, adaptation, distribution and reproduction in any medium or format, as long as you give appropriate credit to the original author(s) and the source, provide a link to the Creative Commons license, and indicate if changes were made. The images or other third party material in this article are included in the article's Creative Commons license, unless indicated otherwise in a credit line to the material. If material is not included in the article's Creative Commons license and your intended use is not permitted by statutory regulation or exceeds the permitted use, you will need to obtain permission directly from the copyright holder. To view a copy of this license, visit http://creativecommons. org/licenses/by/4.0/.

\section{References}

1. Nimer SD. Myelodysplastic syndromes. Blood. 2008;111:4841-51.

2. Estey E. Acute myeloid leukemia and myelodysplastic syndromes in older patients. J Clin Oncol. 2007;25:1908-15.

3. Look AT. Molecular pathogenesis of MDS. Hematology Am Soc Hematol Educ Program 2005;2005:156-60.

4. Kristinsson SY, Bjorkholm M, Hultcrantz M, Derolf AR, Landgren O, Goldin LR. Chronic immune stimulation might act as a trigger for the development of acute myeloid leukemia or myelodysplastic syndromes. J Clin Oncol. 2011;29:2897-903.

5. Starczynowski DT, Karsan A. Innate immune signaling in the myelodysplastic syndromes. Hematol Oncol Clin North Am. 2010;24:343-59.

6. Starczynowski DT, Karsan A. Deregulation of innate immune signaling in myelodysplastic syndromes is associated with deletion of chromosome arm 5q. Cell Cycle. 2010;9:855-6.

7. Rhyasen GW, Bolanos L, Fang J, Jerez A, Wunderlich M, Rigolino C, et al. Targeting IRAK1 as a therapeutic approach for myelodysplastic syndrome. Cancer Cell. 2013;24:90-104.

8. Maratheftis CI, Andreakos E, Moutsopoulos HM, Voulgarelis M. Toll-like receptor-4 is up-regulated in hematopoietic progenitor cells and contributes to increased apoptosis in myelodysplastic syndromes. Clin Cancer Res. 2007;13:1154-60.

9. Chen X, Eksioglu EA, Zhou J, Zhang L, Djeu J, Fortenbery N, et al. Induction of myelodysplasia by myeloid-derived suppressor cells. J Clin Invest. 2013;123:4595-611.

10. Basiorka AA, McGraw KL, Eksioglu EA, Chen X, Johnson J, Zhang $\mathrm{L}$, et al. The NLRP3 inflammasome functions as a driver of the myelodysplastic syndrome phenotype. Blood. 2016;128:2960-75.

11. Casey SC, Tong L, Li Y, Do R, Walz S, Fitzgerald KN, et al. MYC regulates the antitumor immune response through CD47 and PD-L1. Science. 2016;352:227-31.

12. Staron MM, Gray SM, Marshall HD, Parish IA, Chen JH, Perry $\mathrm{CJ}$, et al. The transcription factor FoxO1 sustains expression of the inhibitory receptor PD- 1 and survival of antiviral CD8(+) T cells during chronic infection. Immunity. 2014;41:802-14.

13. Jiang $\mathrm{Y}, \mathrm{Li} \mathrm{Y}, \mathrm{Zhu} \mathrm{B}$. T-cell exhaustion in the tumor microenvironment. Cell Death Dis. 2015;6:e1792.

14. Abedin S, Platanias LC. PD1 and PDL1 upregulation and survival after decitabine treatment in lower risk MDS. Leuk Lymphoma. 2017;58:764-5.

15. Yang H, Bueso-Ramos C, DiNardo C, Estecio MR, Davanlou M, Geng QR, et al. Expression of PD-L1, PD-L2, PD-1 and CTLA4 in myelodysplastic syndromes is enhanced by treatment with hypomethylating agents. Leukemia. 2014;28:1280-8.

16. Boddu P, Kantarjian H, Garcia-Manero G, Allison J, Sharma P, Daver N. The emerging role of immune checkpoint based approaches in AML and MDS. Leuk Lymphoma. 2018;59:790-802.

17. Wei S, Chen X, Rocha K, Epling-Burnette PK, Djeu JY, Liu Q, et al. A critical role for phosphatase haplodeficiency in the selective suppression of deletion 5q MDS by lenalidomide. Proc Natl Acad Sci USA. 2009;106:12974-9.

18. Davis AC, Wims M, Spotts GD, Hann SR, Bradley A. A null cmyc mutation causes lethality before 10.5 days of gestation in homozygotes and reduced fertility in heterozygous female mice. Genes Dev. 1993;7:671-82.

19. Baudino TA, McKay C, Pendeville-Samain H, Nilsson JA, Maclean KH, White EL, et al. c-Myc is essential for vasculogenesis and angiogenesis during development and tumor progression. Genes Dev. 2002;16:2530-43. 
20. Trapnell C, Pachter L, Salzberg SL. TopHat: discovering splice junctions with RNA-Seq. Bioinformatics. 2009;25:1105-11.

21. Anders S, Pyl PT, Huber W. HTSeq--a Python framework to work with high-throughput sequencing data. Bioinformatics. 2015;31:166-9.

22. Love MI, Huber W, Anders S. Moderated estimation of fold change and dispersion for RNA-seq data with DESeq2. Genome Biol. 2014;15:550.

23. Subramanian A, Tamayo P, Mootha VK, Mukherjee S, Ebert BL, Gillette MA, et al. Gene set enrichment analysis: a knowledgebased approach for interpreting genome-wide expression profiles. Proc Natl Acad Sci USA. 2005;102:15545-50.

24. Munn DH, Bronte V. Immune suppressive mechanisms in the tumor microenvironment. Curr Opin Immunol. 2016;39:1-6.

25. Qorraj M, Bruns H, Bottcher M, Weigand L, Saul D, Mackensen A, et al. The PD-1/PD-L1 axis contributes to immune metabolic dysfunctions of monocytes in chronic lymphocytic leukemia. Leukemia. 2017;31:470-8.

26. Wei S, Eksioglu EA, Cluzeau T, Chen X, Basiorka AA, Burnette $A$, et al. MDS genetic damage is linked to inflammaging induced bone marrow hyperglycemia. In: 13th International Symposium on Myelodysplastic Syndromes. Washington, DC; 2015.

27. Eksioglu EA, Chen X, Heider KH, Rueter B, McGraw KL, Basiorka AA, et al. Novel therapeutic approach to improve hematopoiesis in low risk MDS by targeting MDSCs with the Fc-engineered CD33 antibody BI 836858. Leukemia 2017;31:2172-80.

28. Schneider RK, Schenone M, Ferreira MV, Kramann R, Joyce CE, Hartigan C, et al. Rps14 haploinsufficiency causes a block in erythroid differentiation mediated by S100A8 and S100A9. Nat Med. 2016;22:288-97.

29. Raaijmakers MH. Myelodysplastic syndromes: revisiting the role of the bone marrow microenvironment in disease pathogenesis. Int J Hematol. 2012;95:17-25.

30. Della Porta MG. Myelodysplastic syndromes and bone marrow microenvironment. Leuk Res. 2011;35:1442-3.

31. Raaijmakers MH, Mukherjee S, Guo S, Zhang S, Kobayashi T, Schoonmaker JA, et al. Bone progenitor dysfunction induces myelodysplasia and secondary leukaemia. Nature 2010;464:852-7.

32. Allampallam K, Shetty V, Mundle S, Dutt D, Kravitz H, Reddy PL, et al. Biological significance of proliferation, apoptosis, cytokines, and monocyte/macrophage cells in bone marrow biopsies of 145 patients with myelodysplastic syndrome. Int J Hematol. 2002;75:289-97.
33. Navas T, Zhou L, Estes M, Haghnazari E, Nguyen AN, Mo Y, et al. Inhibition of p38alpha MAPK disrupts the pathological loop of proinflammatory factor production in the myelodysplastic syndrome bone marrow microenvironment. Leuk Lymphoma. 2008;49:1963-75.

34. Stirewalt DL, Mhyre AJ, Marcondes M, Pogosova-Agadjanyan E, Abbasi N, Radich JP, et al. Tumour necrosis factor-induced gene expression in human marrow stroma: clues to the pathophysiology of MDS? Br J Haematol. 2008;140:444-53.

35. Wang C, Yang Y, Gao S, Chen J, Yu J, Zhang H, et al. Immune dysregulation in myelodysplastic syndrome: Clinical features, pathogenesis and therapeutic strategies. Crit Rev Oncol Hematol. 2018;122:123-32.

36. Zambetti NA, Ping Z, Chen S, Kenswil KJ, Mylona MA, Sanders MA, et al. Mesenchymal inflammation drives genotoxic stress in hematopoietic stem cells and predicts disease evolution in human pre-leukemia. Cell Stem Cell. 2016;19:613-27.

37. Cluzeau T, McGraw KL, Irvine B, Masala E, Ades L, Basiorka AA, et al. Pro-inflammatory proteins S100A9 and tumor necrosis factor-alpha suppress erythropoietin elaboration in myelodysplastic syndromes. Haematologica. 2017;102:2015-20.

38. Chocarro-Calvo A, Garcia-Martinez JM, Ardila-Gonzalez S, De la Vieja A, Garcia-Jimenez C. Glucose-induced beta-catenin acetylation enhances Wnt signaling in cancer. Mol Cell. 2013;49:474-86.

39. He TC, Sparks AB, Rago C, Hermeking H, Zawel L, da Costa LT, et al. Identification of c-MYC as a target of the APC pathway. Science. 1998;281:1509-12.

40. Dokun OY, Florl AR, Seifert HH, Wolff I, Schulz WA. Relationship of SNCG, S100A4, S100A9 and LCN2 gene expression and DNA methylation in bladder cancer. Int J Cancer. 2008;123:2798-807.

41. List AF, Sun Z, Verma A, Bennett JM, McGraw KL, Nardelli LA, et al. Combined Treatment with Lenalidomide (LEN) and Epoetin Alfa (EA) Is Superior to Lenalidomide Alone in Patients with Erythropoietin (Epo)-Refractory, Lower Risk (LR) NonDeletion 5q [Del(5q)] Myelodysplastic Syndrome (MDS): Results of the E2905 Intergroup Study-an ECOG-ACRIN Cancer Research Group Study, Grant CA180820, and the National Cancer Institute of the National Institutes of Health. Blood. 2016;128:223-223.

42. Socolovsky M, Fallon AE, Wang S, Brugnara C, Lodish HF. Fetal anemia and apoptosis of red cell progenitors in Stat5a-/-5b-/mice: a direct role for Stat5 in $\mathrm{Bcl}-\mathrm{X}(\mathrm{L})$ induction. Cell. 1999;98:181-91. 